\title{
Los discursos de la pandemia. Nuevas estrategias de comunicación del riesgo en un nuevo contexto sociocultural
}

\author{
The pandemic discourses. New risk communication strategies \\ in a new sociocultural context*
}

JAVIER NESPEREIRA GARCÍA

UNIVERSIDAD DE VALLADOLID

Recibido: 02/04/2014

Aceptado: 25/05/2014

ABSTRACT: This paper proposes a rhetorical-discourse framework for the analysis of public health crisis. The last epidemic crisis have highlighted the problematical character of definitions of health risk, illness/disease, health, and scientific authority, as discursive social constructions in a global communication context increasingly complex. Using the influenza A (H1N1) pandemic crisis in 2009-2010 as a model, I put forward the main discourse genres involved in the construction of the pandemic as a new social event, and its potential interdiscursive ties. Its connections with the rhetorical-argumentative strategies of the public health authorities in the crisis communication management has been also underlined.

Keywords: risk communication, discourse genres, rhetorical-argumentative strategies, influenza $\mathrm{A}(\mathrm{H} 1 \mathrm{~N} 1)$ pandemic.

RESUMEN: El presente trabajo plantea el análisis de las crisis sanitarias desde una perspectiva retórico-discursiva. Las recientes crisis epidémicas han puesto de nuevo en evidencia el carácter problemático del concepto de riesgo sanitario, así como de la noción de enfermedad, de salud, y de autoridad científica, en cuanto construcciones discursivas sociales, en un contexto de comunicación global cada vez más complejo.

(*) Trabajo realizado en el marco del proyecto ministerial de investigación «Retórica constructivista: discursos de la identidad» (FFI2013-40934-R). 
Tomando como ejemplo la crisis provocada por la pandemia de gripe A (H1N1) en 2009 y 2010, exponemos los géneros discursivos más importantes en la construcción de la pandemia como un nuevo fenómeno social, y sus posibles relaciones interdiscursivas, así como su relación con las estrategias retórico-argumentativas de las autoridades sanitarias en la gestión de la comunicación de crisis.

Palabras clave: comunicación de riesgo, géneros discursivos, estrategias retóricoargumentativas, pandemia de gripe A (H1N1).

\section{Introducción}

Las enfermedades epidémicas han constituido un importante elemento de transformación en los procesos históricos, de manera que las grandes crisis epidémicas han sido, al mismo tiempo, crisis históricas sociales (McNeill, 1976: 257). Han supuesto alteraciones de la organización social, cambios en la estructura demográfica, y en los patrones de comportamiento, pero al mismo tiempo han dado lugar a cambios significativos en los valores morales, en la identidad cultural y en la conceptualización del otro, como han puesto de manifiesto autores como Michel Foucault (2009) y Susan Sontag (2008), entre otros. Estos procesos de transformación cultural y social han sido mediados por numerosos discursos científicos, políticos, religiosos, mediáticos y artísticos que han construido históricamente los significados de la salud, la enfermedad y la medicina, pero también las interpretaciones de conceptos como la culpa, el castigo o la identidad individual y social. En las próximas páginas intentaremos ofrecer una caracterización de los géneros discursivos que participan en las crisis epidémicas como acontecimientos históricos en la sociedad actual.

En la primera década del siglo XXI se han sucedido una serie de alertas sanitarias provocadas por brotes epidémicos de infecciones virales con una distribución global. Entre 2002 y 2003, la neumonía atípica o SARS, causada por un coronavirus, afectó principalmente a algunos países en Asia y a Canadá, aunque el establecimiento de medidas de prevención tuvo consecuencias sociales y económicas en todo el mundo. Entre 2004 y 2006, la gripe aviar, provocada por un influenzavirus del serotipo $\mathrm{H} 5 \mathrm{~N} 1$, fue objeto de una importante alerta sanitaria internacional al comprobarse su contagio ocasional a seres humanos en forma de infección grave. La Organización Mundial de la Salud 
(en adelante oms) señaló la posibilidad de que una cepa de un influenzavirus animal se adaptase a la especie humana gracias a una modificación genética, lo cual podría originar una pandemia.

En abril de 2009, un brote de gripe en México, provocado por un nuevo serotipo de Influenzavirus, llevó a la OMS a declarar el estado de alerta sanitaria. La rápida propagación de la epidemia a otros estados americanos, en especial a EE. UU., y a Europa, así como la verificación de su elevada capacidad de contagio entre humanos, comportó la declaración por la directora general de la OMs, Margaret Chan, del nivel 6 de alerta sanitaria, o fase pandémica, el 11 de junio. Catorce meses más tarde, el 10 de agosto de 2010, la oms declaraba la conclusión de la fase 6 de la alerta de influenza pandémica, y el inicio de un período post-pandémico.

La OMS, como autoridad sanitaria internacional, ha adquirido en esta última década un renovado protagonismo institucional y mediático en virtud de su papel decisivo en la gestión de estas crisis epidémicas mundiales. Junto a la OMS, otros organismos, como el Center for Disease Control and Prevention de Atlanta en EE. UU. (en adelante CDC), se han convertido en instituciones científicas de referencia tanto para profesionales de la salud pública como para los ciudadanos no expertos, gracias a su presencia en los medios de comunicación como fuentes de información.

Sin embargo, este protagonismo se ha visto empañado por las dudas surgidas con respecto a su gestión de la última crisis epidémica, provocada por el nuevo virus de la gripe, que dio lugar a la primera pandemia del siglo XXI. Expertos epidemiólogos y virólogos, algunos vinculados a la oMs como asesores científicos, así como periodistas especializados en salud pública, denunciaron la existencia de un conflicto de intereses entre la autoridad sanitaria internacional y las empresas farmacéuticas encargadas de la producción de antivirales y vacunas pandémicas (Cohen y Carter, 2010; Godlee, 2010; Flynn, 2010; Keil et al., 2011).

Por último, es necesario situar estas crisis epidémicas en un contexto social internacional que, desde finales de la última década del siglo $\mathrm{xx}$, ha estado marcado por dos circunstancias muy distintas. Por un lado, una pérdida muy significativa de la credibilidad y la confianza en las instituciones sanitarias y científicas a raíz de los errores de gestión y de comunicación de crisis sanitarias alimentarias como la de las «vacas locas» (en adelante BSE/vCJD) entre 1996 y 2001, una crisis de confianza que se ha verificado especialmente en Europa (Millstone y Van Zwanenberg, 2000). Por otro lado, una situación de alarma internacional desencadenada por los atentados del 11 de septiembre de 2001 en Nueva York, a los cuales siguió una alerta sanitaria bioterrorista por infección con esporas de Bacillus anthracis, y que ha hecho del riesgo epidémico una 
variable de la lucha contra el terrorismo y del escenario geopolítico, de manera particular en la sociedad estadounidense (Schoch-Spana, 2004).

\section{La pandemia como crisis de comunicación}

Las crisis sanitarias epidémicas a las que acabamos de referirnos han sido al mismo tiempo crisis sociales con un importante componente mediático (Gil Calvo, 2009; Bernardo y Pellisser, 2009). Asimismo, durante la última crisis, causada por la pandemia de gripe A (H1N1) entre 2009 y 2010, la información circulante por Internet a través de las redes sociales desplazó a los medios de comunicación tradicionales como fuente de información principal, desestabilizando las relaciones entre la opinión pública y las autoridades sanitarias (Semir y Revuelta, 2010). Si consideramos que Internet se ha convertido en los últimos años en la principal fuente de información sobre temas relacionados con la salud (Katz, Rico y Acord, 2006; Camacho Markina, 2010), cabe suponer y esperar que esta dilución tanto del papel del periodista especializado en salud (Semir y Revuelta, 2010) como de la autoridad científica de las instituciones sanitarias y sus expertos (Kroll-Smith, 2003: 638) debida a Internet y al auge de las redes sociales caracterizará las próximas crisis sanitarias.

Los escenarios, los canales y los actores de la comunicación del riesgo sanitario son ahora mucho más complejos, y las diferentes interpretaciones de un mismo acontecimiento pueden reproducirse, transformarse y transmitirse nuevamente con mucha mayor rapidez que hace apenas una década. Esta circunstancia ha llevado a algunos autores a cuestionarse cómo se construyen en este nuevo contexto comunicativo las representaciones sociales del conocimiento epidemiológico y médico-sanitario. A este respecto, Kroll-Smith habla de una difusa autoridad retórica, en buena medida de naturaleza mediática, la cual, en el discurso sanitario, comparte contexto de comunicación con la autoridad institucional (Kroll-Smith, 2003: 628).

Desde esta perspectiva, el estudio de la comunicación del riesgo, y en particular de la gestión de la comunicación de nuevos riesgos epidémicos, debe llevarse a cabo en el ámbito de marcos multidisciplinares que den cuenta de la complejidad discursiva del riesgo sanitario como construcción social y cultural. La investigación de la percepción del riesgo bajo el enfoque racionalista de la psicología cognitiva (Slovic, 1987, entre otros trabajos de este autor y sus colaboradores) y su análisis del efecto de los medios de comunicación en la amplificación social del riesgo (por ejemplo, Kasperson et al., 1988), han demostrado su operatividad en el estudio de riesgos conocidos. Sin embargo, 
presentan serias limitaciones frente a riesgos caracterizados por la falta de conocimiento científico, o bien cuando no existe consenso entre los expertos en cuanto a sus consecuencias y su aceptabilidad (Douglas, 1990: 9-10). Este ha sido el caso de las recientes epidemias y pandemias, en las cuales el componente especulativo y probabilístico en la valoración del riesgo ha cobrado especial importancia, al tiempo que se planteaban escenarios catastróficos. En la actual sociedad global, de la comunicación y de la información, en la que permanentemente se cuestiona el papel de la ciencia y la tecnología como autoridad en la toma de decisiones sobre el riesgo, este refuerza su papel como objeto de controversia política y social, como «recurso forense» (Douglas, 1990). El debate sobre riesgo sanitario y sobre salud pública se convierte, por tanto, en un debate ideológico, como en el caso de la epidemia de VIH/SIDA (Douglas, 1990: 12 y ss.).

Por su parte, otros autores han destacado la diferente naturaleza de un mismo riesgo en diferentes contextos socioculturales. En este sentido, por su propia definición, el riesgo solo existe como representación (Beck, 2006: 215; Van Loon, 2000: 166), como el resultado de una ecuación en la que sus diferentes términos pueden a su vez ser incógnitas que dependan de otras ecuaciones. De este modo, el riesgo, y en particular un nuevo riesgo, constituye lo que Law denomina un «objeto virtual», es decir, un objeto creado en su mismo proceso de representación (Law, 1996: 298). Para Van Loon, desde el ámbito de la ciencia y la tecnología, el riesgo como objeto virtual se introduce en la realidad social a través de diferentes mecanismos retóricos y géneros discursivos que establecen su significado en un determinado contexto (Van Loon, 2002: 57).

En los últimos años, las diferentes teorías sociales sobre la salud pública, la enfermedad y la medicina, así como sobre el riesgo, han coincidido en destacar la naturaleza ideológica y discursiva de estos fenómenos sociales, y cómo este hecho debe tenerse en cuenta en el estudio de la comunicación del riesgo sanitario y de su gestión (Radley, 1994; Radley y Billig, 1996; Lupton, 1999: 16; Lupton, 2003: 19-21; Zinn, 2010). La crisis de la gripe A (H1N1) en 2009 y 2010, y la controversia sobre su gestión, ha tenido lugar en un contexto social, cultural y de comunicación en el que esta naturaleza ideológica y discursiva del riesgo ha quedado aún más de manifiesto. A continuación, y siguiendo el caso de la primera pandemia del siglo XXI como modelo por su complejidad discursiva, esbozaremos una posible caracterización de los diferentes géneros discursivos que participan de la construcción de las nuevas crisis sanitarias, así como las posibles interrelaciones entre discursos de diferentes géneros. 


\section{Géneros retórico-argumentativos. La pandemia como objeto de debate político y social}

Con la crisis de la gripe A ( $1 \mathrm{~N} 1)$ los conceptos de «pandemia» y de «riesgo pandémico» adquirieron una importante dimensión política y social desde el momento de la comunicación del brote de la nueva influenza en México y EE. UU., y su propagación a Europa, a finales de abril de 2009. La posibilidad de que se declarase una pandemia de gripe implicaba la activación de los contratos para el suministro de antivirales y fabricación de vacunas, firmados por la mayor parte de los estados más desarrollados con algunas empresas farmacéuticas, siguiendo las recomendaciones de la OMS tras la crisis de la gripe aviar en 2005. La incertidumbre y la falta de consenso científico sobre la posible gravedad de la nueva epidemia, y el enorme gasto económico que comportaba la aplicación de dichos contratos en un contexto de grave crisis económica, dieron lugar a un intenso debate sobre los límites de la definición de pandemia establecida por la OMS.

El origen de este debate radica en los planes de preparación y respuesta frente a pandemias elaborados por la OMS en 2005 y en 2009, en los cuales se propuso la nueva definición que permitió la declaración de pandemia en junio de 2009. Las principales críticas contra la oms se basaban en que los nuevos criterios establecidos en el documento de orientación de 2009 permitían que un proceso epidémico global de escasa gravedad pudiera ser declarado como pandemia (Cohen y Carter, 2010), causando un gasto desproporcionado de recursos económicos si se aplicaban las recomendaciones de la OMS. De este modo, podríamos considerar estos documentos de orientación de la OMS, así como otros documentos similares producidos por otras instituciones sanitarias de referencia como los $\mathrm{CDC}$, como discursos con una clara finalidad retórica. Es decir, se trata de discursos producidos con la intención de persuadir a las autoridades políticas de que una determinada definición de pandemia como hecho problemático, tanto por su carácter incierto como por sus consecuencias, es la definición que más se ajusta a la realidad, y que, por lo tanto, las actuaciones recomendadas son útiles y necesarias.

Compartimos así con Majone la idea de que el análisis de la formulación de políticas ambientales, energéticas o sanitarias debe hacerse desde un complejo marco argumentativo y retórico que supla las limitaciones del mero conocimiento científico y técnico (Majone, 1997: 57-59). Por lo tanto, desde nuestro punto de vista, la clasificación retórica de los géneros discursivos podría ayudarnos a caracterizar algunos de los discursos institucionales que 
construyen el acontecimiento pandémico y sus interpretaciones sociales, en la sociedad actual.

\subsection{Género deliberativo: la construcción discursiva del nuevo riesgo}

El modelo de discurso deliberativo es el discurso político, pronunciado ante una asamblea, en el cual el orador trata de persuadir a su auditorio de lo útil o lo inútil de la acción futura que se aconseja o desaconseja llevar a cabo. El género deliberativo es posiblemente el más importante en el debate social sobre el riesgo, como noción en virtud de la cual se toman decisiones basadas en especulaciones sobre acontecimientos futuros. En la elaboración de las políticas de gestión del riesgo sanitario se lleva a cabo un proceso consultivo y deliberativo del que participan expertos científicos, los agentes sociales implicados e interesados -los grupos de presión que defienden intereses de los ciudadanos, pero también aquellos que defienden los intereses económicos de empresas médicas o de la industria farmacéutica-, y, por supuesto, los responsables políticos encargados de debatir y redactar las leyes que regulan la política sanitaria.

Los documentos de orientación de la oms para la preparación y respuesta frente a pandemias constituyen un ejemplo de este género discursivo. Como hemos apuntado, la finalidad de estos documentos técnicos es persuadir de la utilidad de las medidas de prevención propuestas en cada fase de una posible pandemia (WHO, 2005: 3; wHO, 2009: 45). Al mismo tiempo, estas actuaciones son presentadas no solo como útiles, sino también honestas, cuyos objetivos últimos son morales, como favorecer la coordinación, la cooperación y la comunicación entre las instituciones, o reforzar el liderazgo de las autoridades políticas.

Asimismo, el carácter dilemático de la noción de riesgo se pone de manifiesto explícitamente en estos discursos de la OMS, por lo que a las cualidades de lo útil y lo honesto, hay que añadir lo necesario de las medidas recomendadas por la OMS para contrastar o limitar las graves consecuencias de una pandemia. De este modo, concurren en estos documentos técnicos de la autoridad sanitaria internacional las tres cualidades principales del discurso deliberativo o suasorio clásico (Lausberg, 1966: §§ 233-235). Igualmente, en tanto que discursos deliberativos, las pruebas que sostienen argumentativamente los planes de actuación de la OMS se basan principalmente en ejemplos de anteriores epidemias y pandemias, como las gripes de 1918, 1957 y 1968, el SARS o la gripe aviar. Estos ejemplos permiten, por un lado, reforzar la argumentación por las 
consecuencias que hacen útiles y necesarias las nuevas medidas, mientras que, por otro lado, refuerzan la autoridad moral de la propia institución sanitaria.

En este sentido, es en este género retórico en el que la autoridad del emisor del discurso es más importante, una cuestión especialmente crítica cuando el discurso tiene un auditorio no versado en la materia que constituye la causa (Pujante, 1999: 85), como con frecuencia ocurre en el debate sobre el riesgo cuyo conocimiento científico es escaso y/o controvertido. A este respecto, la relevancia de la autoridad moral de quien elabora y emite el discurso nos remite al papel del experto científico y de la autoridad sanitaria en la comunicación del riesgo epidémico, y a la importancia de la confianza del auditorio en el orador para que su discurso logre su objetivo. En este caso impulsar una determinada política sanitaria de alerta frente a pandemias.

\subsection{Género judicial: la construcción de la interpretación moral de las nuevas epidemias}

Las crisis sanitarias, mientras suceden o una vez transcurridas, también dan lugar a discursos judiciales o forenses, en los cuales bien las autoridades sanitarias, bien las autoridades políticas, bien determinados sectores de la industria - por ejemplo la industria médica o farmacéutica- son acusados de errores en la valoración del riesgo o en su gestión, y deben defenderse de estas acusaciones. Para algunos teóricos sociales del riesgo (Beck et al., 1997), la existencia de esta confrontación dialéctica y reflexiva entre los intereses científicos, tecnológicos e institucionales y los intereses del resto de la sociedad es uno de los elementos que caracterizan la actual «sociedad del riesgo global» como resultado de la «modernidad reflexiva».

Como ya hemos apuntado, la crisis de la gripe A (H1N1) dio lugar a un intenso debate público sobre el posible conflicto de intereses en las recomendaciones de la OMS y en su gestión de la pandemia, ya que algunos de sus asesores científicos epidemiólogos y virólogos podrían haber sido, al mismo tiempo, colaboradores y asesores de las empresas farmacéuticas encargadas de la fabricación de antivirales y las denominadas «vacunas pandémicas». Un requerimiento firmado por el doctor Wolfgang Wodarg y otros parlamentarios del Consejo de Europa en diciembre de 2009, llevó a convocar sendas audiencias públicas en la Asamblea Parlamentaria del Consejo de Europa en enero y en abril de 2010 (Flynn, 2010).

En ese mismo año, en el mes de junio, se publicaba en la edición digital del British Medical Journal (en adelante $B M J$ ) un artículo de investigación que 
presentaba pruebas consistentes de la existencia de un grave conflicto de intereses en la gestión de la pandemia de gripe A (H1N1) (Cohen y Carter, 2010), acompañado del editorial de una de las redactoras del $B M J$ en el que se criticaba duramente el trabajo de la principal autoridad sanitaria internacional (Godlee, 2010). Pocos días después, la página web de la oms publicaba una carta a la redacción del $B M J$, firmada por la doctora Margaret Chan, directora general de la OMS, donde refutaba las acusaciones vertidas contra la institución.

Este debate, similar al que tiene lugar con respecto a otros riesgos sanitarios marcados por la incertidumbre como el de la crisis de la BSE/VCDJ o sobre los posibles riesgos derivados del consumo de alimentos genéticamente modificados, es posiblemente más susceptible a un análisis retórico-argumentativo como discursos judiciales de acusación y refutación, que a un análisis científico-técnico. En el caso de la problemática gestión de la pandemia de nueva gripe, tanto el discurso del epidemiólogo Wolfgang Wodarg como el artículo y el editorial publicados en el $B M J$ fundamentan su argumentación en las contradicciones en las que ha incurrido la OMS, restando importancia a los datos epidemiológicos que permitieron declarar la pandemia, y amplificando el conflicto de intereses y la falta de transparencia en las decisiones y actuaciones de la autoridad sanitaria internacional, apelando a las emociones de la opinión pública.

A su vez, los representantes de la OMS tampoco recurren a argumentos científico-técnicos sólidos, y fundamentan su refutación de las acusaciones en el argumento de la autoridad del organismo internacional como institución de referencia en salud pública, y en la apelación a las emociones de la opinión pública. En este sentido, sus discursos de defensa parecen adoptar más bien la forma de discursos laudatorios de la organización que representan, limitándose a negar las acusaciones sin aportar datos o hechos que puedan rebatirlas.

\section{Géneros narrativos. La pandemia como reelaboración del mito de la peste}

En las últimas décadas, numerosos autores han puesto en evidencia la importancia de los géneros narrativos en la construcción discursiva de la enfermedad (Hydén, 1997, para una revisión bibliográfica a este respecto). Principalmente desde el ámbito de la psicología social y las representaciones sociales, se ha prestado especial interés a la construcción y significación narrativa llevada a cabo por las personas afectadas por enfermedades crónicas, enfermedades mentales, depresión o estrés (cf. por ejemplo Herzlich, 1973: 91 y ss.; Radley, 1994: 136 y ss.). 
Otros autores se han ocupado de la relación entre las narrativas, en particular de las narrativas de ficción, y los riesgos epidémicos como fenómenos sociales (Tomes, 2000; 2002; Foertsch, 2001; Nerlich, Hamilton y Rowe, 2002; Tomes, 2002; Nerlich, 2004; Ostherr, 2005; Nerlich y James, 2009). Estas investigaciones demuestran la importancia de los relatos de ficción en la construcción de modelos cognitivos para las crisis epidémicas, en cuanto representaciones de los mecanismos mediante los cuales acontece un evento de estas características. Constituyen así modelos de actuación de los diferentes protagonistas en estos hechos, tanto ciudadanos como las propias instituciones sanitarias o políticas. Al mismo tiempo establecen una determinada relación causal entre los hechos, y entre las acciones de los personajes y sus consecuencias, expresando de forma más o menos explícita una determinada interpretación ideológica y una valoración moral.

A este respecto, en las dos décadas a caballo entre el siglo XX y el siglo XXI ha tenido lugar un auge significativo de la ficción de temática epidémica, en particular en los géneros de ciencia-ficción y de terror. La mayor parte de estos relatos se sustentan en un marco narrativo apocalíptico o post-apocalíptico muy presente en las manifestaciones culturales de la sociedad actual (cf., entre otros, Berger, 1999; Cordle, 2008), y que a su vez constituyen expresiones de una realidad cultural propia del cambio de siglo (Žižek, 2012). El marco narrativo de una pandemia de dimensiones catastróficas ha permitido la revitalización, a principios del siglo XXI, de un género de ficción en decadencia como el género zombi, que actualmente goza de una enorme popularidad en todo tipo de soportes narrativos -literatura impresa y digital, cine, cómic y videojuegos(Fuentes, 2012), así como de la atención especial de algunos estudios culturales y filosóficos (cf., entre otros: Pérez Rufi, 2009; Martínez Lucena, 2010; Fernández Gonzalo, 2011; Martínez Lucena, 2012).

Resulta significativo, desde nuestro punto de vista, que la revitalización de estos géneros narrativos coincida con el período de frecuentes crisis sanitarias, alimentarias y principalmente epidémicas, al que nos hemos referido en las páginas anteriores, desde la alarma por la BSE/vCDJ entre 1996 y 2001, hasta la reciente crisis pandémica de 2009 y 2010. Estos nuevos relatos de ficción reelaboran y amplifican el mito de la «peste» como castigo social, tal y como señalaba Susan Sontag en su ensayo sobre la conceptualización de la epidemia del VIH/SIDA (Sontag, 2008: 151 y ss.).

Sin embargo, a diferencia de la gran pandemia de finales del siglo $\mathrm{xx}$, la significación moral de las nuevas epidemias no parece estar vinculada, como el VIH/SIDA o la sífilis, a su transmisión sexual (Sontag, 2008: 169 y ss.) ni a la sociedad internacional de bloques enfrentados fruto de la Guerra Fría 
(Foertsch, 2001). La nueva ficción epidémica y catastrofista ha cambiado la noción de peligro, asociado al pecado y a la contaminación, en virtud de la cual un individuo contaminado amenazaba a la sociedad, por la noción postmoderna del riesgo, según la cual la sociedad y sus acciones suponen una amenaza para el individuo, tal y como adelantara Mary Douglas al referirse a una sociedad occidental cada vez más individualista (Douglas 1990: 7). Esta sociedad individualista es la que reflejarían, por ejemplo, las obras del exitoso género zombi.

\section{Las relaciones entre géneros discursivos en la comunicación de crisis epidémicas. El ejemplo de un discurso didáctico}

La temática narrativa catastrofista apocalíptica o post-apocalíptica constituye en sí misma un atractivo esquema narrativo que favorece su reproducción en diferentes discursos (Borchardt, 1990: 2; Kermode, 2000: 95-96). En esta línea, desde nuestro punto de vista, el esquema de fases propuesto en los documentos de preparación y respuesta frente a pandemias de la OMS conforma un modelo narrativo que conduce a la pandemia como final inevitable. Epidemiológicamente, a partir del plan de 2005 y de forma aún más evidente desde el nuevo documento de orientación de 2009, el escenario internacional queda definido en términos pandémicos. Es decir, o nos encontramos dirigiéndonos irremisiblemente hacia una pandemia -fases de 1 a 5-, o bien nos encontramos sufriendo las consecuencias de una pandemia -fase 6-, o bien, como en la actualidad, nos encontramos en una situación definida como «pospandémica», de retorno a la fase 1 prepandémica. Este planteamiento establece discursivamente un marco de permanente alerta, en consonancia con otros marcos del discurso político contemporáneo dominante, como el de la lucha contra el terrorismo (Morales y Pujante, 2009, entre otros).

Por otro lado, la ficción epidémica incorpora los discursos institucionales y de las autoridades sanitarias. Las declaraciones de un experto científico o de un representante de las autoridades en un medio de comunicación es un recurso narrativo omnipresente en toda la ficción epidémica contemporánea, que permite proporcionar información sobre los hechos al espectador al tiempo que confiere verosimilitud al relato. Asimismo, la forma y la veracidad con la que se muestran estos discursos institucionales informativos construyen ideológicamente el papel de dichas instituciones en ese tipo de acontecimientos.

La representación gráfica del agente patógeno causante de la pandemia y de la propia pandemia contribuye asimismo a la verosimilitud del relato de ficción mediante la introducción y transformación de elementos propios del 
discurso científico y de la representación del conocimiento científico, como imágenes de microscopio electrónico, secuencias genéticas o mapas de distribución y propagación de la enfermedad. Estos elementos permiten a su vez representar conceptos y objetos invisibles a los sentidos, como los virus o la misma noción de contagio. Al convertirse en símbolos e iconos en una secuencia narrativa, estos elementos gráficos adoptan una determinada interpretación ideológica (Ostherr, 2005).

La hibridación genérica conforma, entonces, el complejo escenario de la comunicación actual, en el cual un discurso con una finalidad concreta contiene elementos lingüísticos, gráficos, audiovisuales de diferente naturaleza. Estos son recibidos e interpretados de manera no lineal y, en muchas ocasiones, parcial, y por individuos o grupos sociales de muy diferentes niveles de conocimiento previo sobre un determinado acontecimiento. Un ejemplo significativo, desde nuestro punto de vista, es la campaña de divulgación de preparación frente a catástrofes naturales puesta en marcha por el Office of Public Health Preparedness and Response del CDC en 2011. ${ }^{1}$ El material informativo y didáctico de esta campaña incluye un extenso apartado denominado «Zombi Preparedness», ${ }^{2}$ en el que la narrativa de ficción pandémica del género de terror zombi en forma de relato gráfico y otro contenido multimedia sirve de plataforma para el discurso institucional deliberativo, por el que se trata de persuadir a la opinión pública de la utilidad y la necesidad de la adopción de determinadas medidas.

\section{Conclusiones}

Las recientes crisis sanitarias globales, como la provocada por la pandemia de gripe A (H1N1), han agravado la crisis de credibilidad y de confianza en las instituciones sanitarias y en el conocimiento científico-técnico. El complejo contexto de la comunicación en la sociedad actual ha modificado radicalmente la noción de autoridad, un concepto fundamental para entender las relaciones discursivas e ideológicas entre la construcción del conocimiento científico experto y la construcción del conocimiento no experto. En este escenario, las autoridades sanitarias han transformado profundamente sus estrategias de comunicación, en un proceso de hibridación y de convergencia con el discurso no científico.

1. http://www.cdc.gov/phpr/areweprepared.htm (01/03/2014).

2. http://www.cdc.gov/phpr/zombies.htm (01/03/2014). 
La finalidad de esta transformación es claramente retórico-persuasiva: modificar el comportamiento de los individuos y los grupos sociales para evitar situaciones de riesgo sanitario o conseguir un estilo de vida más saludable. Sin embargo, como todo discurso de construcción y transmisión del conocimiento, el discurso científico de las instituciones sanitarias es un discurso marcadamente ideológico que construye determinados significados, creencias, actitudes y normas públicas, necesarias para el ejercicio y el mantenimiento del poder (Van Dijk, 2009).

Con el ejemplo de la crisis de la gripe A (H1N1) hemos evidenciado la importancia de los diferentes géneros discursivos en las estrategias argumentativas y de comunicación. El análisis de las relaciones entre géneros argumentativos y géneros narrativos, y de su incorporación a otros géneros, como el discurso didáctico, el discurso mediático, o incluso al propio discurso científico, constituye una interesante perspectiva para el estudio discursivo de las crisis sanitarias como complejas crisis de comunicación. Los resultados de estas investigaciones contribuirían así a contrastar la actual crisis de confianza en las instituciones, que, en el ámbito de la salud pública, puede comportar un grave perjuicio para el conjunto de la sociedad, especialmente en situaciones de crisis sanitaria.

\section{Referencias bibliográficas}

BECK, U. (2006 [1999]): La sociedad del riesgo global, 2. ${ }^{\text {a }}$ ed., Madrid, Siglo XXI.

BECK, U. et al. (1997): Modernizacion reflexiva. Politica, tradicion y estética en el orden social moderno, Madrid, Alianza.

Berger, J. (1999): After the End. Representations of Post-Apocalypse, Minneapolis, University of Minnesota Press.

Camacho Markina, I. (2010). «Noticias sobre salud y medicina: mucho más que información» en Camacho Markina, I. (ed.): La especialización en el periodismo. Formarse para informar, Sevilla/Zamora, Comunicación Social, 141-162.

Cohen, D.; Carter, P. (2010): «Who and the pandemic flu "conspiracies"», British Medical Journal, 340: c2912.

CORDLE, D. (2008): States of suspense. The nuclear age, postmodernism and United States fiction and prose, Manchester-Nueva York, Manchester University Press.

Douglas, M. (1990): «Risk as a Forensic Resource», Doedalus, 119 (4): 1-16.

Fernández Gonzalo, J. (2011): Filosofía zombi, Barcelona, Anagrama. 
FLYNN, P. (2010): The handling of the H1N1 pandemic: more transparency needed. 2010, <http://assembly.coe.int/CommitteeDocs/2010/20100604_ H1n1pandemic_E.pdf> [Consulta: 01-12-2012].

Foertsch, J. (2001): Enemies within. The Cold War and the AIDS crisis in literature, film, and culture, Urbana and Chicago, University of Illinois Press.

Foucault, M. (2009 [1979]). El nacimiento de la biopolítica, Madrid, Akal.

Gil Calvo, E. (2009): Crisis crónica. La construcción social de la gran recesión, Madrid, Alianza.

GodLEe, F. (2010): «Conflicts of interest and pandemic flu», British Medical Journal, 340: c2947.

HydÉN, L.-C. (1997): «Illness and narrative», Sociology of Health \& Illness, 19 (1): 48-69.

KASPERSON, R. et al., (1988): «The Social Amplification of Risk: A Conceptual Framework». Risk Analysis, 8 (2): 177-187.

KeIL, U. et al., (2011): "The invention of the swine-flu pandemic», European Journal of Epidemiology, 26: 187-190.

Kermode, F. (2000): El sentido de un final. Estudios sobre la teoría de la ficción, Barcelona, Gedisa.

Kroll-Smith, S. (2003): «Popular media and "excessive daytime sleepiness": a study of rhetorical authority in medical sociology», Sociology of Health \& Illness, 25 (6): 625-643.

LAw, J. (1996): «Organizing accountabilities: ontology and the mode of accounting» en Munro, R.; J. Mouritsen (eds.): Accountability. Power, Ethos and the Technologies of Managing, Londres-Boston, Boston International Thomson Business Press, 283-306.

Lupton, D. (2003 [1994]): Medicine as Culture. Illness, Disease and the Body in Western Societies, 2. ${ }^{\text {e }}$ ed., Londres, SAGE.

MaJone, G. (1997 [1989]): Evidencia argumentación y persuasión en la formulación de políticas, México DF, Fondo de Cultura Económica.

Martínez Lucena, J. (2012). Ensayo Z. Una explicación filosófica del fenómeno zombi y de su éxito como metáfora de nuestro tiempo, Córdoba, Berenice.

Millstone, E.; P. Van Zwanenberg (2000): «A crisis of trust: For science, scientist or for institution?», Nature Medicine, 6 (12): 1307-1308.

NerLICH, B. (2004): «War on foot and mouth disease in the UK, 2001: Towards a cultural understanding of agriculture», Agriculture and Human Values, 21 (1): $15-25$. 
Nerlich, B.; R. James (2009): ««The post-antibiotic apocalypse» and the «war on superbugs»: Catastrophe discourse in microbiology, its rhetorical form and political function», Public Understanding of Science, 18 (5): 574-590.

OSTHERR, K. (2005): Cinematic prophylaxis. Globalization and contagion in the discourse of world health, Durham-Londres, Duke University Press.

Pujante, D. (1999): El hijo de la persuasión. Quintiliano y el estatuto retórico, 2. ${ }^{\text {a }}$ ed., Logroño, Instituto de Estudios Riojanos.

Pujante, D.; E. Morales (2009): «Una actuación política contra la opinión de la ciudadanía: El último discurso de Aznar en el Parlamento español justificando la guerra de Irak (diciembre de 2003)», Discurso \& Sociedad, 3 (4): 769-806.

Radley, A. (1994): Making Sense of Illness. The Social Psychology of health and disease, Londres, SAGE.

Sсносн-Spana, M. (2004): «Bioterrorism. US public heatlh and a secular apocalypse», Anthropology Today, 20 (5): 8-13.

SLovic, P. (1987): «Perception of Risk», Science, 236 (4799): 280-285.

SonTaG, S. (2008): La enfermedad y sus metáforas. El sida y sus metáforas, Barcelona, Mondadori.

Tomes, N. (2000): «The making of a germ panic, then and now», American Journal of Public Health, 90 (2): 191-198.

VAn DiJK, T. A. (2009): Discurso y poder, Barcelona, Gedisa.

VAN Loon, J. (2002): Risk and technological culture. Towards a sociology of virulence, Londres, Routledge.

Wно (2005): Plan mundial de la oms de preparación para una pandemia de influenza. Funciones y recomendaciones de la oms para las medidas nacionales, <http://www.who.int/csr/resources/publications/influenza/wHO_ CDS_CSR_GIP_2005_5sP.pdf $>$ [Consulta: 01-12-2012].

- (2009): Preparación y respuesta ante una pandemia de influenza. Documento de Orientación de la OMS, <http://www.who.int/csr/swine_flu/ Preparacion_Pand_ESP.pdf $>$ [Consulta: 01-12-2012].

ZINN, J. O. (2010): «Risk as Discourse: Interdisciplinary Perspectives», CADAAD: Critical Approaches to Discourse Analysis Across Disciplines, 4 (2): 106-124.

ŽıžEK, S. (2012): Viviendo en el final de los tiempos, Madrid, Akal. 